\title{
An Improved Electrical Switching and Phase-Transition Model for Scanning Probe Phase-Change Memory
}

\author{
Lei Wang, Si-Di Gong, Jing Wen, and Ci Hui Yang \\ School of Information Engineering, Nanchang Hangkong University, Nanchang 330063, China \\ Correspondence should be addressed to Lei Wang; leiwang@nchu.edu.cn
}

Received 26 October 2015; Accepted 27 December 2015

Academic Editor: Thierry Baron

Copyright ( 2016 Lei Wang et al. This is an open access article distributed under the Creative Commons Attribution License, which permits unrestricted use, distribution, and reproduction in any medium, provided the original work is properly cited.

\begin{abstract}
Scanning probe phase-change memory (SPPCM) has been widely considered as one of the most promising candidates for nextgeneration data storage devices due to its fast switching time, low power consumption, and potential for ultra-high density. Development of a comprehensive model able to accurately describe all the physical processes involved in SPPCM operations is therefore vital to provide researchers with an effective route for device optimization. In this paper, we introduce a pseudo-threedimensional model to simulate the electrothermal and phase-transition phenomena observed during the SPPCM writing process by simultaneously solving Laplace's equation to model the electrical process, the classical heat transfer equation, and a rate equation to model phase transitions. The crystalline bit region of a typical probe system and the resulting current-voltage curve obtained from simulations of the writing process showed good agreement with experimental results obtained under an equivalent configuration, demonstrating the validity of the proposed model.
\end{abstract}

\section{Introduction}

Digital data is ubiquitous in the modern world and is intimately linked to nearly all facets of the daily lives of citizens. The advancing prevalence of digital data has caused the amount of global data created and copied in digital form to double every two years and is expected to reach 44 Zettabytes by 2020 [1]. To avoid a corresponding increase in the actual physical volume required to meet this unprecedented growth rate a significant increase in the storage capacity of memory devices is required. Unfortunately, the marginal opportunities remaining for improving the capacity of conventional storage devices are severely restricted by their inherent physical limits [2]. For this reason, various emerging storage technologies have been proposed. From these, scanning probe phase-change memory (SPPCM), as illustrated in Figure 1, has been considered to be one of the most promising contenders.

Binary data is represented in SPPCM according to the varying resistance of phase-change materials, in particular, $\mathrm{Ge}_{2} \mathrm{Sb}_{2} \mathrm{~T}_{5}$ (GST). The fact that the resistance of GST can be switched between a highly resistive state (amorphous phase) and a highly conductive state (crystalline phase) by injecting a current through a nanoscale scanning probe endows SPPCM with several merits including ultra-high recording density and high reading contrast [2-4].

Due to the advantageous features of SPPCM over conventional storage, SPPCM has recently received tremendous attention from researchers worldwide. However, the majority of research efforts have been dedicated to realizing resistance switching experimentally [5-7]. As a result, a detailed theoretical framework that can account for the electrothermal and phase-transition phenomena occurring inside SPPCM remains unfulfilled. To the best of our knowledge, a theoretical model of SPPCM processes has been reported only by a single group of researchers (Wright's group at the University of Exeter) [2]. In Wright's model, the physical mechanism of SPPCM was regarded as a combination of electrical, thermal, and phase transformation processes. However, some relatively simple assumptions were introduced into the descriptions of the electrical and thermal conductivities of GST, which severely limited the accuracy of the established model. Furthermore, the phase transformation phenomenon in Wright's model was governed by the Johnson-MehlAvrami-Kolmogorov (JMAK) law which exhibits an Arrhenius crystallization mode that is contradictory to recent 


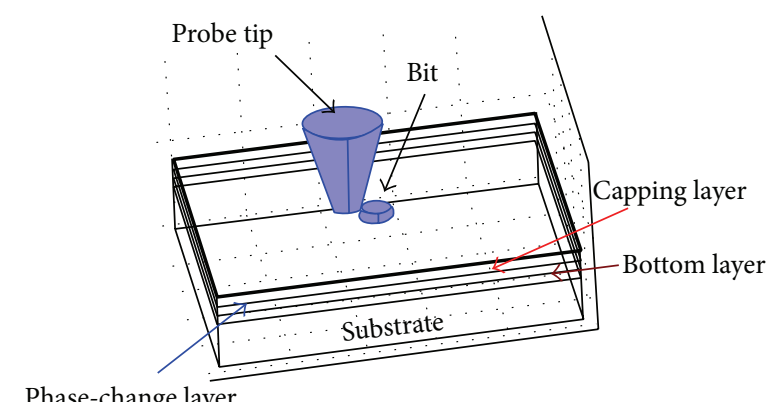

FIGURE 1: Structure of a scanning probe phase-change memory device.

experimental findings clearly indicating a non-Arrhenius behavior [8]. Therefore, it is necessary to develop a more physically realistic model representative of all the physical processes of SPPCM to aid researchers in obtaining a better understanding of the resulting SPPCM performances and, ultimately, to achieve optimal system design.

\section{Simulation Strategy}

The physical mechanisms that determine the write performance of SPPCM using a GST medium involve electrical, thermal, and phase transformation processes. Laplace's equation is adopted here to model the electrical process, which, because it is similar to the model proposed by Wright's group, is not described in detail here. The only difference between Wright's electrical model and that presently proposed arises from the electrical conductivity of the amorphous GST. In Wright's model, the electrical conductivity of the amorphous GST is simply defined as a function of the electric field. This assumption however neglects influences owing to semiconductor defects and energy traps that affect electrical transport mechanism of chalcogenide glasses [9] and limits the accuracy of the obtained resistive switching behavior of the GST. To mitigate this limitation, the electrical conductivity of the amorphous GST ( $\left.\sigma_{\mathrm{am}}\right)$ in this paper is assumed to be governed by a Poole-Frenkel (PF) mechanism [10], which is given by

$$
\sigma_{\mathrm{am}}=\left(\frac{2 k_{B} T}{q j_{0} \Delta z} \exp \left(\frac{E_{A}}{k_{B} T}\right) \operatorname{csch}\left(\frac{q F \Delta z}{2 k_{B} T}\right)\right)^{-1},
$$

where $F$ is the electric field, $\Delta z$ is the average distance between localized states with a value of $3.3 \mathrm{~nm}, E_{A}$ is the activation energy for PF conduction with a value of $0.2 \mathrm{eV}$, $k_{B}$ is Boltzmann's constant, $q$ is the electron charge, $j_{0}$ is the integral of the trap distribution in the gap above the Fermi level with a value of $10^{25} \mathrm{~m}^{-3}$, and $T$ is the temperature. The electrical conductivity of the crystalline GST $\left(\sigma_{\text {cryst }}\right)$ here is considered to be dependent on temperature alone [2] and is given as

$$
\sigma_{\text {cryst }}=\sigma_{0 \text { cryst }} \exp \left(-\frac{E_{C}}{k_{B} T}\right)
$$

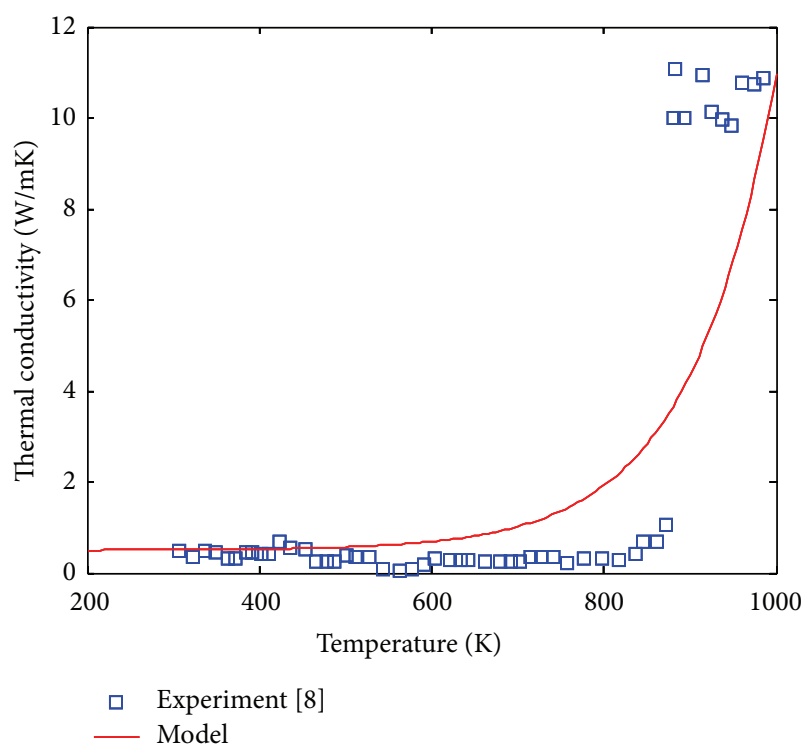

FIgURE 2: Previously reported thermal conductivity of crystalline GST as a function of temperature [8].

where $\sigma_{0 \text { cryst }}$ is a constant with a value of $1.5 \times 10^{4} \Omega^{-1} \mathrm{~m}^{-1}$ and $E_{C}$ is the activation energy for crystallization with a value of $2.6 \mathrm{eV}$.

Solving Laplace's equation gives rise to the electric field distribution inside the GST medium that can be used as the heat source for the subsequent thermal model. In this case, the classical heat transfer equation is introduced to account for the temperature distribution inside the GST medium. However, the thermal conductivity of the crystalline GST usually exhibits temperature dependence [8], and we here introduce that dependence using a power law according to (3), as described in Figure 2. Consider

$$
K_{\text {cryst }}=5 \times 10^{-4} \exp \left(\frac{T}{100}\right)+0.4
$$

where $K_{\text {cryst }}$ is the thermal conductivity of the crystalline GST.

Note that Figure 2 shows an accurate reproduction of a steep increase on the experimental $K_{\text {cryst }}$ around $900 \mathrm{~K}$ as well as a less close match on the experimental $K_{\text {cryst }}$ above $900 \mathrm{~K}$. This is probably due to the values of chosen parameters adopted for (3). More importantly, as the main concern of this paper is to model the transition of the GST from the amorphous state to the crystalline state, it is therefore necessary to more closely imitate the variation of the $K_{\text {cryst }}$ below $900 \mathrm{~K}$ (i.e., crystalline regime) than the case with temperature above $900 \mathrm{~K}$ (i.e., liquid regime).

The generated temperature distribution is then deployed to determine the extent of phase transformation using the phase-change model. We here employ a rate equation for evaluating the phase-change process to overcome the aforementioned drawbacks of the JMAK law. The rate equation 
enables the calculation of the crystal fraction of the GST medium $\left(f_{x}\right)$ according to

$$
\frac{d f_{x}}{d t}=\left(1-f_{x}\right) k_{x}
$$

where $k_{x}$ is the thermal-activated kinetic constant, denoted as

$$
k_{x}=\left(t_{x 1} \exp \left(\frac{E_{x 1}}{k_{B} T}\right)+t_{x 2} \exp \left(\frac{E_{x 2}}{k_{B} T}\right)\right)^{-1},
$$

where $t_{x 1}$ and $t_{x 2}$ are preexponential factors with values of $1.5 \times 10^{-29} \mathrm{~s}$ and $1 \times 10^{-14} \mathrm{~s}$, respectively, and $E_{x 1}$ and $E_{x 2}$ are activation energies with values of $2.9 \mathrm{eV}$ and $1.1 \mathrm{eV}$, respectively. Based on the descriptions presented thus far, the value of $f_{x}$, when subjected to an applied current, can be determined by simultaneously solving Laplace's equation, the heat transfer equation, and the rate equation. Note that, for the GST medium, the electrical conductivity $\left(\sigma_{\mathrm{GST}}\right)$ and thermal conductivity $\left(K_{\text {cryst }}\right)$ should vary along with the growth of $f_{x}$. These relationships are described in our model as follows:

$$
\begin{gathered}
\sigma_{\mathrm{GST}}=\sigma_{\mathrm{am}}\left(1-f_{x}\right)+\sigma_{\text {cryst }} f_{x}, \\
K_{\mathrm{GST}}=K_{\mathrm{am}}\left(1-f_{x}\right)+K_{\text {cryst }} f_{x} .
\end{gathered}
$$

Here $K_{\mathrm{am}}$ is the thermal conductivity of amorphous GST with a value of 0.2 and $K_{\text {cryst }}$ can be obtained directly from (3). During the simulation, an electric pulse and ground potential are applied to the electrical probe and bottom electrode, respectively. Cylindrical symmetry is implemented to reduce a fully three-dimensional problem into one of only two dimensions. The calculations were all performed using COMSOL MULTIPHYSICS ${ }^{\mathrm{TM}}$.

\section{Results and Discussion}

A typical phase-change media stack with a platinum-slicide (PtSi) tip was previously proposed to optimize the writing performance of SPPCM, where a $10 \mathrm{~nm}$ thick GST layer was sandwiched between a $2 \mathrm{~nm}$ thick diamond-like carbon (DLC) capping layer and a $40 \mathrm{~nm}$ thick titanium nitride (TiN) bottom layer [11]. To verify the physical validity of the developed model, we first performed a physical experiment involving the aforementioned structure to record a crystalline bit within the surrounding amorphous region using a write pulse of $2.6 \mathrm{~V}$ and a $1 \mu$ s duration and the surface was subsequently scanned using a readout voltage of $1 \mathrm{~V}$. Owing to the remarkable difference between the resistances of the amorphous and crystalline phases, the readout current obtained with the tip in contact with the crystalline region significantly differs from that when the tip scanned over the amorphous region. Consequently, by measuring the readout current, the boundary of the resulting crystalline bit could be readily determined. As can be seen from Figure 3(a), a single crystalline bit with a diameter of around $100 \mathrm{~nm}$ was generated and the surface exhibits good electrical (current) contrast with the surrounding amorphous region. In addition to examination of the crystalline bit, we also measured the current-voltage $(I-V)$ curve obtained during the application of the writing pulse using a $15 \mathrm{k} \Omega$ series resistor, as shown in Figure 3(c). The figure clearly indicates that the writing current remains very nearly zero at the early stage of the writing pulse and exhibits a very slow increase owing to the high resistivity of the amorphous phase. Once the voltage increased to around $1.8 \mathrm{~V}$, however, a sudden increase in the writing current corresponding with a sudden decrease in voltage associated with a standard voltage snapback behavior was observed, which can be explained by the well known threshold switching effect $[12,13]$. Here, the resistance of the GST medium was considerably reduced when the threshold switching voltage was achieved, and the voltage across the series resistor began to exceed that across the SPPCM, leading to the observed snapback shape. The consequently large writing current induced crystallization, which further reduced the system resistance. As a result, the writing current undergoes a continuous increase until the applied pulse reaches its peak value.

The simulated crystalline bit and $I-V$ curve conducted under the same conditions as those employed in the experiment are also shown in Figure 3. According to Figure 3(b), a continuous crystalline bit approximately $100 \mathrm{~nm}$ in diameter was formed during the simulation, showing good agreement with the experimental observation, given in Figure 3(a). I-V characteristics resulting from our newly developed model as well as those obtained using Wright's model are presented in Figure 3(c) along with the experimentally obtained values. It is observed that both models exhibit similar maximum current values, while the proposed model provides a threshold voltage very close to the experimentally observed value in addition to the characteristic snapback shape, which however cannot be replicated by Wright's model. Note that it is difficult to reproduce exactly the results reported by the actual experiment due to uncertainty associated with establishing suitable values for the chosen physical parameters [14]. However, as observed in Figure 3(c), our model provides a reasonably accurate description of the threshold switching behaviour of an actual SPPCM device, whereas the previous model is unable to replicate the snapback shape of real devices. Therefore, it would be beneficial to introduce this model into future SPPCM device simulations for either the writing or the readout processes.

\section{Conclusions}

An improved numerical model was presented to describe the phase-transition behavior of a GST medium for scanning probe storage applications. The proposed model consisting of Laplace's equation to model the electrical process, the classical heat transfer equation, and a rate equation for predicting phase transition was able to predict the extent of phase transformation and the electrical switching behavior of a GST medium when subjected to an electrical pulse. The simulation results were in good agreement with experimental findings, which validates the physical reality of the model. 


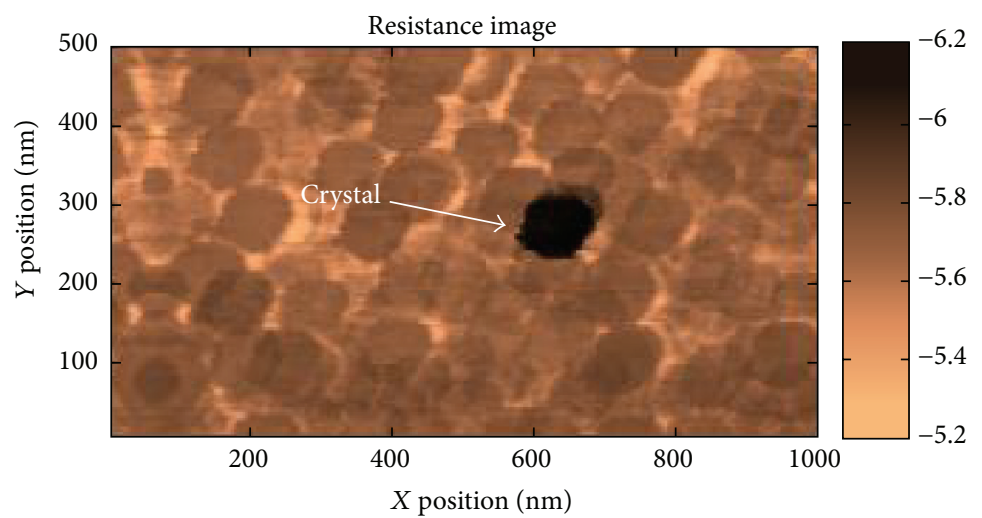

(a)

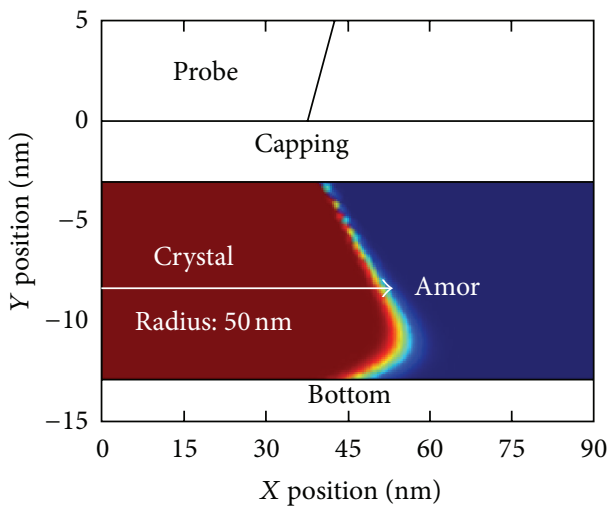

(b)

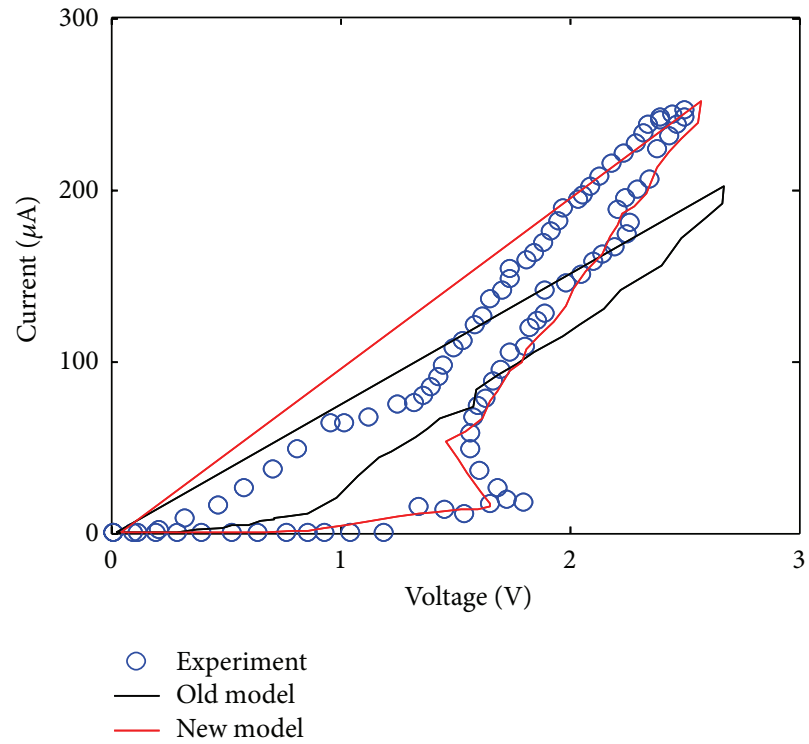

(c)

Figure 3: The crystalline bit region obtained from a typical probe system using a $2.6 \mathrm{~V}$ pulse of $1 \mu$ s duration: (a) by experiment; (b) by simulation based on the proposed model; and (c) by the corresponding $I$ - $V$ characteristics obtained during the application of the writing pulse, which compares the experimental results with those obtained from the newly proposed model and that proposed by Wright et al. [2].

\section{Competing Interests}

The authors declare that they have no competing interests.

\section{Acknowledgments}

The authors acknowledge the financial support of the National Natural Science Foundation of China (nos. 61201439 and 61402218).

\section{References}

[1] L. Wang, C. D. Wright, M. M. Aziz, P. Shah, C.-H. Yang, and G.-W. Yang, "Terabit-per-square-inch scanning probe phasechange memory model based on nucleation-growth theory," Materials Letters, vol. 112, pp. 51-53, 2013.

[2] C. D. Wright, M. Armand, and M. M. Aziz, "Terabit-per-squareinch data storage using phase-change media and scanning electrical nanoprobes," IEEE Transactions on Nanotechnology, vol. 5, no. 1, pp. 50-61, 2006.

[3] L. Wang, C. D. Wright, M. M. Aziz, C.-H. Yang, and G.-W. Yang, "A physics-based three dimensional readout model for phasechange probe memory," Current Applied Physics, vol. 14, no. 9, pp. 1296-1300, 2014.

[4] L. Wang, C. D. Wright, M. M. Aziz, J. Yin, and G.-W. Yang, "A physics-based three dimensional model for write and read performances of phase-change probe memory," Journal of Nanoscience and Nanotechnology, vol. 15, no. 4, pp. 2785-2789, 2015.

[5] S. Gidon, O. Lemonnier, B. Rolland, O. Bichet, C. Dressler, and Y. Samson, "Electrical probe storage using Joule heating in phase change media," Applied Physics Letters, vol. 85, no. 26, p. 6392, 2004.

[6] H. Bhaskaran, A. Sebastian, A. Pauza, H. Pozidis, and M. Despont, "Nanoscale phase transformation in $\mathrm{Ge}_{2} \mathrm{Sb}_{2} \mathrm{Te}_{5}$ using encapsulated scanning probes and retraction force microscopy," 
Review of Scientific Instruments, vol. 80, no. 8, Article ID 083701, 2009.

[7] H. Satoh, K. Sugawara, and K. Tanaka, "Nanoscale phase changes in crystalline $\mathrm{Ge}_{2} \mathrm{Sb}_{2} \mathrm{Te}_{5}$ films using scanning probe microscopes," Journal of Applied Physics, vol. 99, no. 2, Article ID 024306, 2006.

[8] N. Ciocchini, M. Cassinerio, D. Fugazza, and D. Ielmini, "Evidence for non-arrhenius kinetics of crystallization in phase change memory devices," IEEE Transactions on Electron Devices, vol. 60, no. 11, pp. 3767-3774, 2013.

[9] Q. Cao and P. Liu, "Crystalline-amorphous phase transition of hyperbranched polyurethane phase change materials for energy storage," Journal of Materials Science, vol. 42, no. 14, pp. 56615665, 2007.

[10] D. Ielmini, “Threshold switching mechanism by high-field energy gain in the hopping transport of chalcogenide glasses," Physical Review B, vol. 78, no. 3, Article ID 035308, 2008.

[11] L. Wang and S. Gai, "The next generation mass storage devices-physical principles and current status," Contemporary Physics, vol. 55, no. 2, pp. 75-93, 2014.

[12] M. Cassinerio, N. Ciocchini, and D. Ielmini, "Evidence for electrically induced drift of threshold voltage in $\mathrm{Ge}_{2} \mathrm{Sb}_{2} \mathrm{Te}_{5}$," Physics Letters, vol. 103, no. 2, Article ID 023502, 2013.

[13] A. Redaelli, A. Pirovano, A. Benvenuti, and A. L. Lacaita, "Threshold switching and phase transition numerical models for phase change memory simulations," Journal of Applied Physics, vol. 103, Article ID 111101, 2008.

[14] Z. Fan and D. E. Laughlin, "Three dimensional crystallization simulation and recording layer thickness effect in phase change optical recording," Japanese Journal of Applied Physics, vol. 42, no. 2, article 800, 2003. 

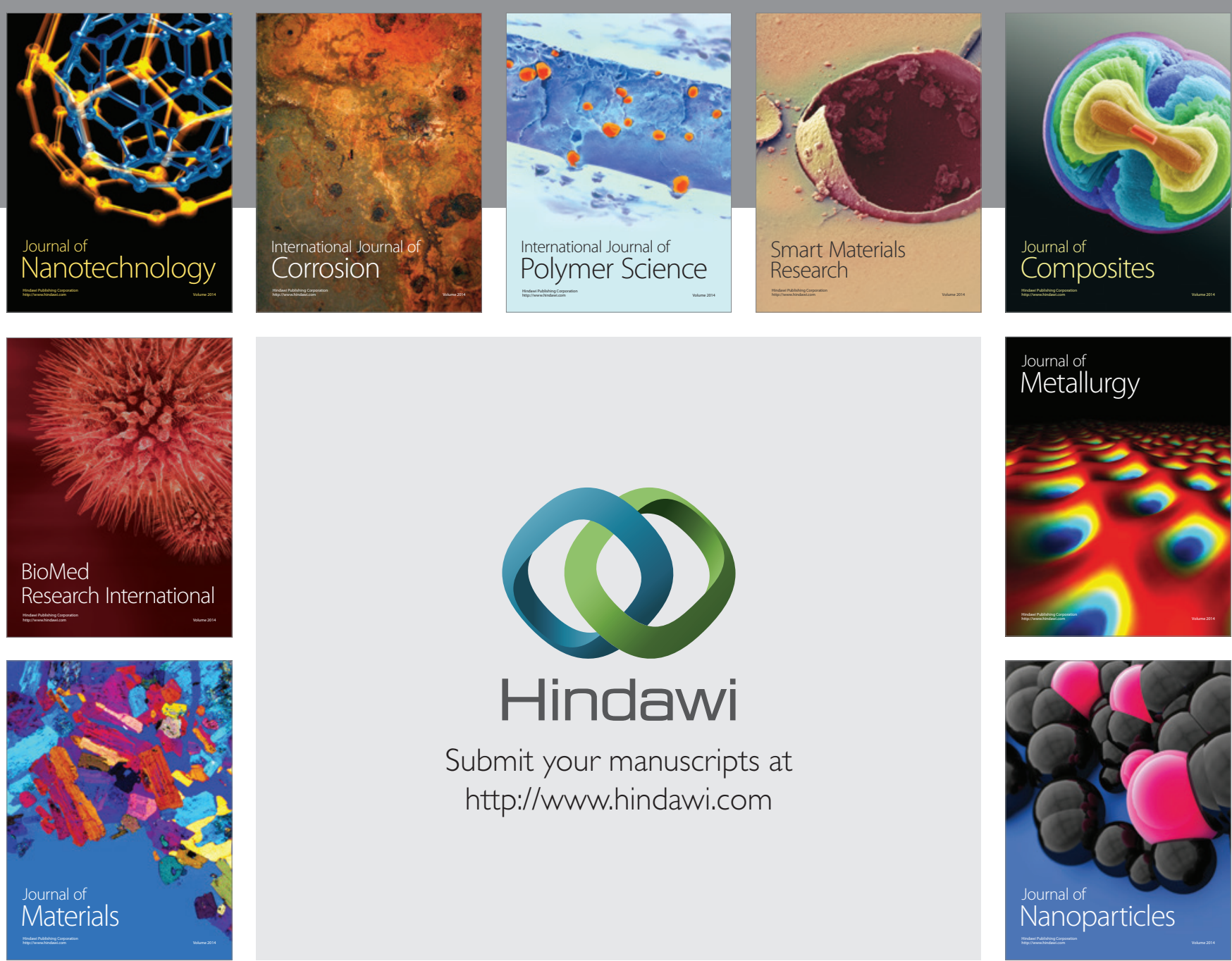

\section{Hindawi}

Submit your manuscripts at

http://www.hindawi.com

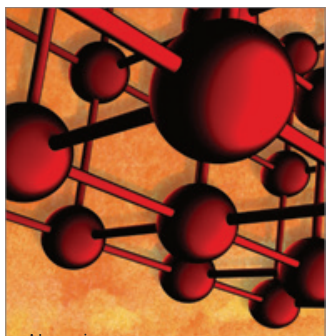

Materials Science and Engineering
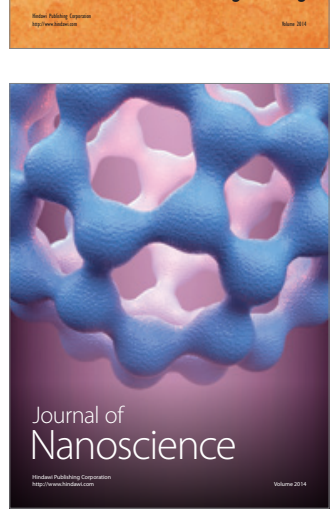
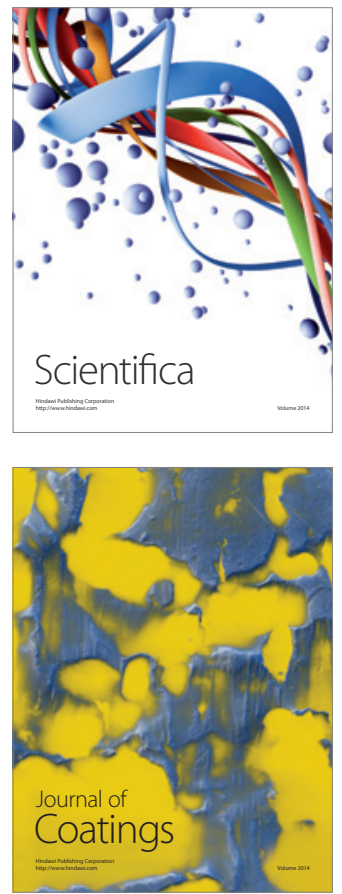
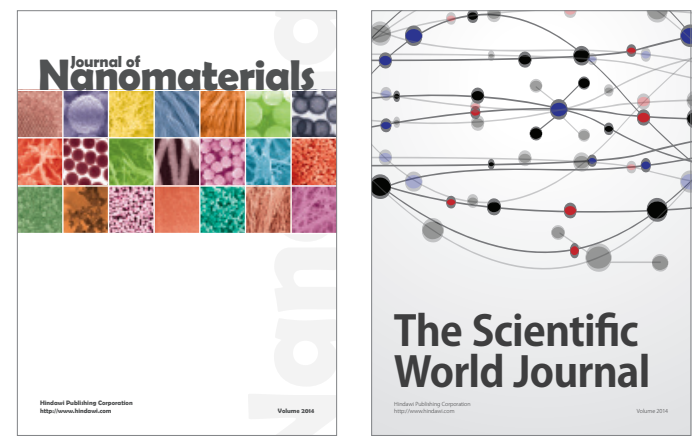

The Scientific World Journal
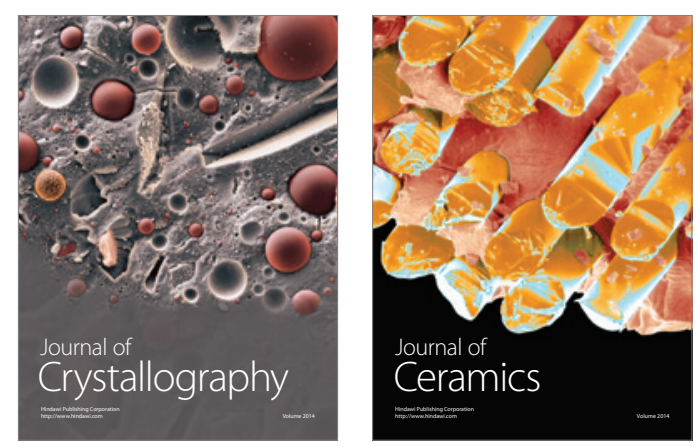
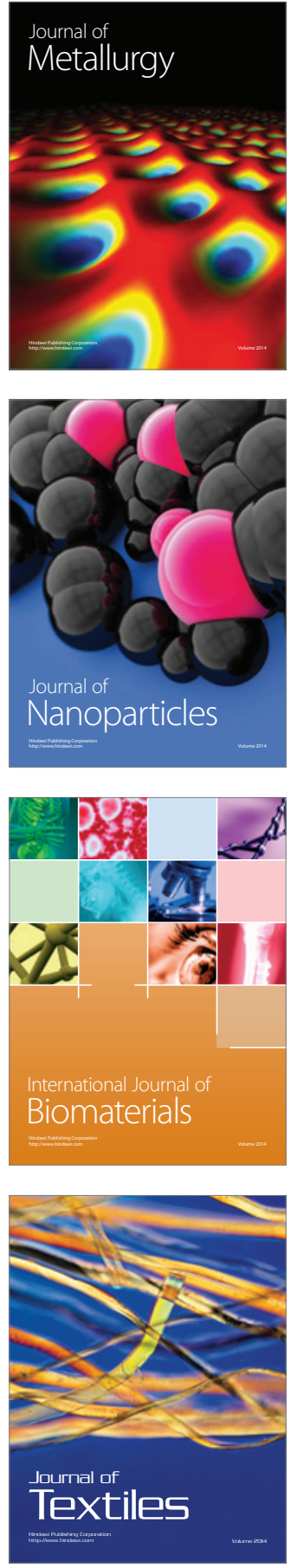